\title{
SOLUTION OF INVERSE DYNAMICS OF STEWART-GOUGH PLATFORM USING SUBSTRUCTURE TECHNIQUE
}

\author{
TRAN XUAN TIEN \\ Hung Yen University of Technology and Education, Hung Yen, Viet Nam \\ e-mail: tranxuantienck@utehy.edu.vn \\ DAVID CIRKL \\ Technical University of Liberec, Liberec, Czech Republic \\ e-mail: david.cirkl@tul.cz
}

\begin{abstract}
The inverse dynamics method is applied to the investigation of dynamical behavior of a parallel manipulator. The substructure technique is used for creating a differential-algebraic system of equations that describes motion of the Stewart-Gough platform. The system of equations is modified into a set of linear differential equations for the determination of the solution of the inverse dynamics problem. A computational algorithm is developed to solve the inverse dynamics of the manipulator. Several trajectories of the moving platform are simulated as a result of the inverse dynamics problem of the Stewart-Gough platform according to the substructure technique.
\end{abstract}

Keywords: inverse dynamic, Stewart-Gough platform, parallel manipulation, substructure technique

\section{Introduction}

A parallel manipulator is a mechanical system using several serial chains to support a single platform or an end-effector. This type of structure provides several benefits to the system, such as higher load capacity, higher stiffness and a more precise execution of complex end-effector motion. For this reason, it is applied in various applications in precise manufacturing, medical science and in space exploration equipment. One of the best-known parallel manipulators, which consist of six linear actuators, is known as the Stewart-Gough platform. The dynamical analysis of parallel manipulators is complicated because of the existence of multiple closed-loop chains. Lots of approaches have already been proposed, including the Newton-Euler formulation (Do and Yang, 1988; Guglielmetti and Longchamp, 1994; Tsai and Kohli, 1990), Lagrangian formulation (Lebret et al., 1993; Miller and Clavel, 1992; Pang and Shahingpoor, 1994), and the principle of virtual work (Angeles, 1997; Codourey and Burdet, 1997; Zanganeh et al., 1997; Miller, 1995; Zhang and Song, 1993). The task presented in this article is similar to the simulation result of Tsai (2000). However, instead of using the concept of linking Jacobian matrices, this study focuses on the substructure technique. The Stewart-Gough platform is then divided into substructures for kinematic and dynamic analyses of the mechanism. A system of linear differential equations describing motion and mechanical connections of the substructures is created and synthesized into a matrix form. An algorithm for determining the solution to the system of equations is presented. Numerical simulations are performed using Maple software with the input data obtained by Tsai (2000). 


\section{System of equations describing motion of the Stewart-Gough platform}

Following Tsai (2000), the Stewart-Gough platform comprises a circular moving platform connected to a fixed base using six extensible limbs $A_{i} B_{i}(i=1,2, \ldots, 6)$. Each limb is made up of a cylinder (link 1) and piston (link 2), which are connected by a prismatic joint. Link 1 of each limb is connected to the moving platform by a spherical joint at $B_{i}$, while link 2 is connected to the fixed base by a universal joint at $A_{i}$. Each prismatic joint is driven by a hydraulic actuator or DC motor via a linear ball screw. For analysis, we use two coordinate systems as illustrated in Fig. 1. The global coordinate system $\left(O, x_{0}, y_{0}, z_{0}\right)$ is attached to the fixed base and another local coordinate system $(P, u, v, w)$ is attached to the moving platform. Let $d_{i}$ be the length of each limb, $a_{i}$ be the distance from the center of the universal joint $A_{i}$ to the center of mass $O$ of the fixed base and $b_{i}$ be the distance from the center of the spherical joint $B_{i}$ to the center of mass $P$ of the moving platform. For each limb, the masses of the cylinder and piston are represented by $m_{1}$ and $m_{2}$, respectively.

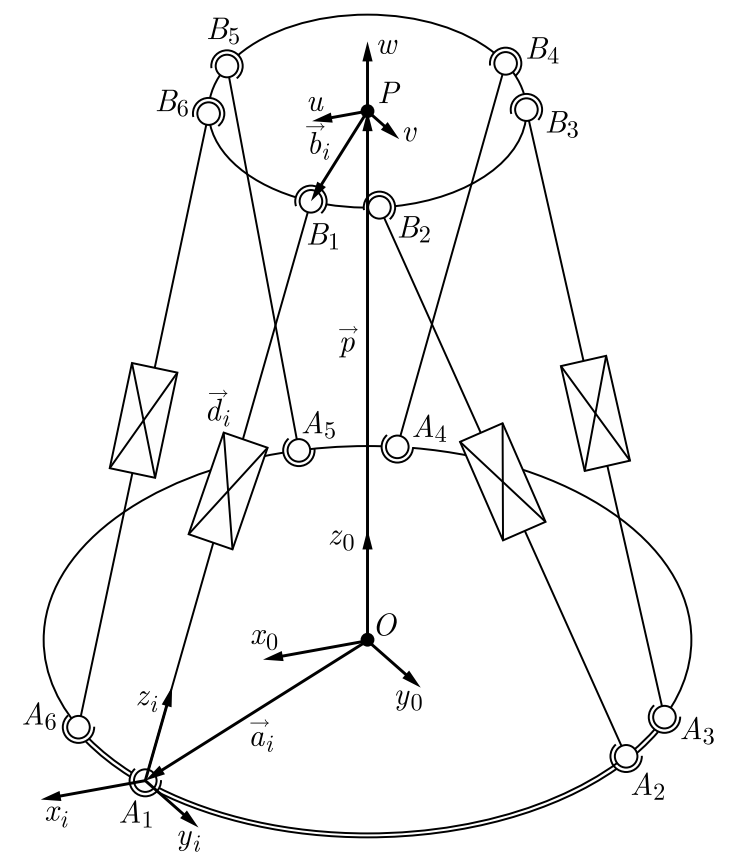

Fig. 1. Schematic of the Stewart-Gough platform

For the sake of convenience, the origin $P$ of the local coordinate system $(P, u, v, w)$ is located in the center of mass of the moving platform. So the position of the moving platform is described in the global coordinate system $\left(O, x_{0}, y_{0}, z_{0}\right)$ by the vector $\mathbf{r}_{P}=\overrightarrow{O P}$. It can be expressed in the form of an algebraic vector as $\mathbf{r}_{P}^{(0)}=\left[x_{P}, y_{P}, z_{P}\right]^{\mathrm{T}}$. The orientation of the moving platform is defined by the orientation of the local coordinate system $(P, u, v, w)$ in the global coordinate system $\left(O, x_{0}, y_{0}, z_{0}\right)$ and is described by the roll-pitch-yaw angles $\left(\varphi_{x}, \varphi_{y}, \varphi_{z}\right)$, and can be expressed by a rotational transformation matrix $\mathbf{A}_{P}$ (see Khang, 2007)

$$
\mathbf{A}_{P}=\left[\begin{array}{ccc}
\mathrm{c} \varphi_{z} \mathrm{c} \varphi_{y} & -\mathrm{s} \varphi_{z} \mathrm{c} \varphi_{x}+\mathrm{c} \varphi_{z} \mathrm{~s} \varphi_{y} \mathrm{~s} \varphi_{x} & \mathrm{~s} \varphi_{z} \mathrm{~s} \varphi_{x}+\mathrm{c} \varphi_{z} \mathrm{~s} \varphi_{y} \mathrm{c} \varphi_{x} \\
\varphi_{z} \mathrm{c} \varphi_{y} & \mathrm{c} \varphi_{z} \mathrm{c} \varphi_{x}+\mathrm{s} \varphi_{z} \mathrm{~s} \varphi_{y} \mathrm{~s} \varphi_{x} & -\mathrm{c} \varphi_{z} \mathrm{~s} \varphi_{x}+\mathrm{s} \varphi_{z} \mathrm{~s} \varphi_{y} \mathrm{c} \varphi_{x} \\
-\mathrm{s} \varphi_{y} & \mathrm{c} \varphi_{y} \mathrm{~s} \varphi_{x} & \mathrm{c} \varphi_{y} \mathrm{c} \varphi_{x}
\end{array}\right]
$$

where $\mathrm{c} \varphi_{i}=\cos \varphi_{i}, \mathrm{~s} \varphi_{i}=\sin \varphi_{i}$, with $i=x, y, z$.

In the local coordinate system $(P, u, v, w)$, the position of the universal joint $B_{i}$ is determined by the vector $\mathbf{b}_{i}=\overrightarrow{P B_{i}}, \mathbf{b}_{i}^{(P)}=\left[b_{i u}, b_{i v}, b_{i w}\right]^{\mathrm{T}}$. 
Each limb is connected to the fixed base by the universal joint $A_{i}$. The $i$-th limb is represented by the local coordinate system $\left(A_{i}, x_{i}, y_{i}, z_{i}\right)$, and its orientation concerning the fixed base can be described by two Euler angles $\left(\phi_{i}, \theta_{i}\right)$. As illustrated in Fig. 2a, the orientation of the coordinate system $\left(A_{i}, x_{i}, y_{i}, z_{i}\right)$ can be considered as a result of the coordinate system $\left(A_{i}, x_{0}^{\prime}, y_{0}^{\prime}, z_{0}^{\prime}\right)$ which is parallel to the coordinate system $\left(O, x_{0}, y_{0}, z_{0}\right)$ and undergoes rotation $\phi_{i}$ about the axis $z_{0}^{\prime}$, resulting in the $\left(A_{i}, x_{0}^{\prime}, y_{0}^{\prime}, z_{0}^{\prime}\right)$ system followed by another rotation by $\theta_{i}$ about the rotary axis $y_{i}^{\prime}$. In this manner, the rotation matrix of the $i$-th limb is given by

$$
\mathbf{A}_{i}=\left[\begin{array}{ccc}
\mathrm{c} \phi_{i} \mathrm{c} \theta_{i} & -\mathrm{s} \phi_{i} & \mathrm{c} \phi_{i} \mathrm{~s} \theta_{i} \\
\mathrm{~s} \phi_{i} \mathrm{c} \theta_{i} & \mathrm{c} \phi_{i} & \mathrm{~s} \phi_{i} \mathrm{~s} \theta_{i} \\
-\mathrm{s} \theta_{i} & 0 & \mathrm{c} \theta_{i}
\end{array}\right]
$$

where $\mathrm{c} \phi_{i}=\cos \phi_{i}, \mathrm{~s} \phi_{i}=\sin \phi_{i}, \mathrm{c} \theta_{i}=\cos \theta_{i}, \mathrm{~s} \theta_{i}=\sin \theta_{i}(i=1, \ldots, 6)$.

(a)

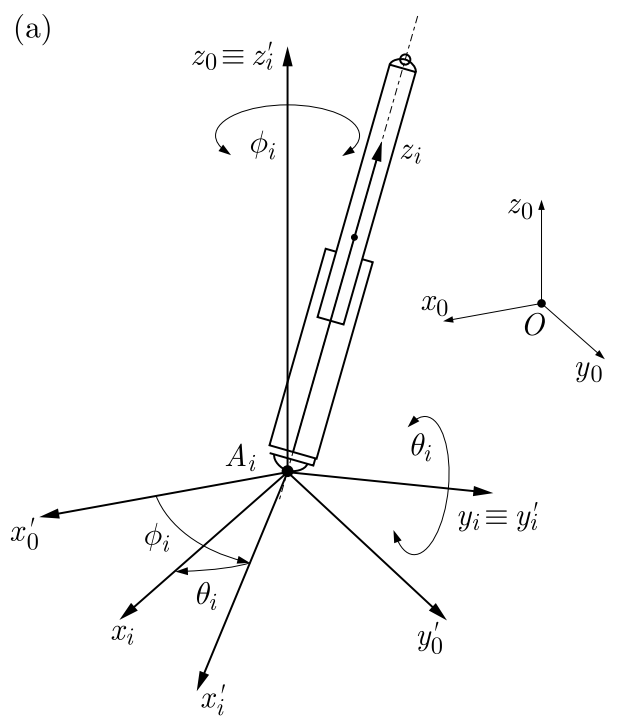

(b)

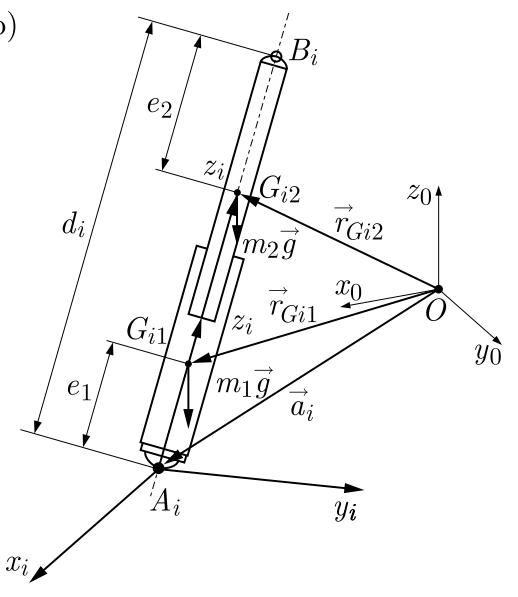

Fig. 2. (a) Two Euler angles of the limb. (b) Free body diagram of a typical limb

The position of the universal joint $A_{i}$ in the global coordinate system $\left(O, x_{0}, y_{0}, z_{0}\right)$ can be described by the algebraic vector $\mathbf{a}_{i}^{(0)}=\left[a_{i x}, a_{i y}, a_{i z}\right]^{\mathrm{T}}$. As illustrated in Fig. $2 \mathrm{~b}$, assume that $e_{1}$ is the distance between $A_{i}$ and the center of mass of the $i$-th cylinder $G_{i 1}$, while $e_{2}$ is the distance between $B_{i}$ and the center of mass of the $i$-th piston. In the local coordinate system $\left(A_{i}, x_{i}, y_{i}, z_{i}\right)$, the algebraic vectors $\mathbf{c}_{1 i}^{(i)}$ and $\mathbf{c}_{2 i}^{(i)}$ represent the positions of the center of mass of the $i$-th cylinder and $i$-th piston, respectively

$$
\mathbf{c}_{1 i}^{(i)}=\left[0,0, e_{1}\right]^{\mathrm{T}} \quad \mathbf{c}_{2 i}^{(i)}=\left[0,0, d_{i}-e_{2}\right]^{\mathrm{T}}
$$

Then, the centers of mass of the cylinder and piston are given by

$$
\mathbf{r}_{G_{i 1}}=\mathbf{a}_{i}+\mathbf{c}_{i 1} \quad \mathbf{r}_{G_{i 2}}=\mathbf{a}_{i}+\mathbf{c}_{i 2}
$$

Thus, in the coordinate system $\left(O, x_{0}, y_{0}, z_{0}\right)$, they are given by the following form

$$
\begin{aligned}
& \mathbf{r}_{G_{i 1}}^{(0)}=\mathbf{a}_{i}^{(0)}+\mathbf{A}_{i} \mathbf{c}_{i 1}^{(i)}=\left[\begin{array}{c}
a_{i x}+\mathrm{c} \phi_{i} \mathrm{~s} \theta_{i} e_{1} \\
a_{i y}+\mathrm{s} \phi_{i} \mathrm{~s} \theta_{i} e_{1} \\
a_{i z}+\mathrm{c} \theta_{i} e_{1}
\end{array}\right] \\
& \mathbf{r}_{G_{i 2}}^{(0)}=\mathbf{a}_{i}^{(0)}+\mathbf{A}_{i} \mathbf{c}_{i 2}^{(i)}=\left[\begin{array}{c}
a_{i x}+\mathrm{c} \phi_{i} \mathrm{~s} \theta_{i}\left(d_{i}-e_{2}\right) \\
a_{i y}+\mathrm{s} \phi_{i} \mathrm{~s} \theta_{i}\left(d_{i}-e_{2}\right) \\
a_{i z}+\mathrm{c} \theta_{i}\left(d_{i}-e_{2}\right)
\end{array}\right]
\end{aligned}
$$


Referring to Fig. 1, the vector loop equation can be written for each limb

$$
\overrightarrow{O A_{i}}+\overrightarrow{A_{i} B_{i}}=\overrightarrow{O P}+\overrightarrow{P B_{i}} \Longleftrightarrow \overrightarrow{a_{i}}+\overrightarrow{d_{i}}=\overrightarrow{r_{P}}+\overrightarrow{b_{i}}
$$

in the form of an algebraic matrix equation

$$
\mathbf{a}_{i}^{(0)}+\mathbf{A}_{i} \mathbf{d}_{i}^{(i)}=\mathbf{r}_{P}^{(0)}+\mathbf{A}_{P} \mathbf{b}_{i}^{(P)}
$$

Following substitution, we obtain

$$
\left[\begin{array}{l}
a_{i x} \\
a_{i y} \\
a_{i z}
\end{array}\right]+\left[\begin{array}{ccc}
\mathrm{c} \phi_{i} \mathrm{c} \theta_{i} & -\mathrm{s} \phi_{i} & \mathrm{c} \phi_{i} \mathrm{~s} \theta_{i} \\
\mathrm{~s} \phi_{i} \mathrm{c} \theta_{i} & \mathrm{c} \phi_{i} & \mathrm{~s} \phi_{i} \mathrm{~s} \theta_{i} \\
-\mathrm{s} \theta_{i} & 0 & \mathrm{c} \theta_{i}
\end{array}\right]\left[\begin{array}{c}
0 \\
0 \\
d_{i}
\end{array}\right]=\left[\begin{array}{c}
x_{P} \\
y_{P} \\
z_{P}
\end{array}\right]+\mathbf{A}_{P}\left[\begin{array}{c}
b_{i u} \\
b_{i v} \\
b_{i w}
\end{array}\right] \quad i=1,2, \ldots, 6
$$

Thus, the position of the $i$-th limb can be determined by a vector of generalized coordinates

$$
\mathbf{q}^{(i)}=\left[\theta_{i}, \phi_{i}, d_{i}\right]^{\mathrm{T}}
$$

By using the substructure technique, the Stewart-Gough platform can be separated into seven substructures consisting of six limbs and a moving platform. Reaction forces act on the $i$-th limb, as illustrated in Fig. 3, where $X_{B i}, Y_{B i}, Z_{B i}$ are the reaction forces at the position of the joint $B_{i}(i=1, \ldots, 6)$ and $\tau_{i}$ is the input force exerted on the $i$-th actuated joint $(i=1, \ldots, 6)$.
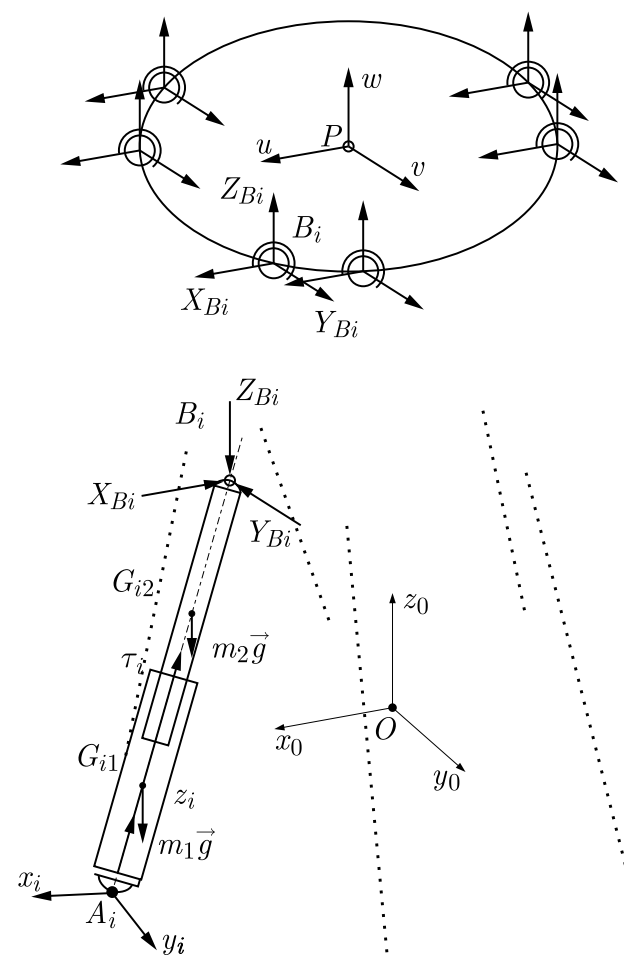

Fig. 3. Free body diagram of substructures

\subsection{System of differential-algebraic equations for motion of each limb}

According to Khang (2007), for each limb, the angular velocity of links 1 and 2, denoted by $\boldsymbol{\omega}_{i 1}$ and $\boldsymbol{\omega}_{i 2}$, respectively, can be calculated using the tilde operator

$$
\widetilde{\mathbf{w}}_{i 1}^{(i)}=\widetilde{\mathbf{w}}_{i 2}^{(i)}=\mathbf{A}_{i}^{\mathrm{T}} \dot{\mathbf{A}}_{i}=\left[\begin{array}{ccc}
0 & -\mathrm{c} \theta_{i} \dot{\phi}_{i} & \dot{\theta}_{i} \\
\mathrm{c} \theta_{i} \dot{\phi}_{i} & 0 & \mathrm{~s} \theta_{i} \dot{\phi}_{i} \\
-\dot{\theta}_{i} & -\mathrm{s} \theta_{i} \dot{\phi}_{i} & 0
\end{array}\right]
$$


Then we get

$$
\mathbf{w}_{i 1}^{(i)}=\mathbf{w}_{i 2}^{(i)}=\left[\begin{array}{c}
-\mathrm{s} \theta_{i} \dot{\phi}_{i} \\
\dot{\theta}_{i} \\
\mathrm{c} \theta_{i} \dot{\phi}_{i}
\end{array}\right]
$$

From (2.4), the Jacobian matrices of translation for the links of the $i$-th limb are given by

$$
\begin{aligned}
\mathbf{J}_{T_{i 1}} & =\frac{\partial \mathbf{r}_{G_{i 1}}^{(0)}}{\partial \mathbf{q}^{(i)}}=\left[\begin{array}{ccc}
\mathrm{c} \phi_{i} \mathrm{c} \theta_{i} e_{1} & -\mathrm{s} \phi_{i} \mathrm{~s} \theta_{i} e_{1} & 0 \\
\mathrm{~s} \phi_{i} \mathrm{c} \theta_{i} e_{1} & \mathrm{c} \phi_{i} \mathrm{~s} \theta_{i} e_{1} & 0 \\
-\mathrm{s} \theta_{i} e_{1} & 0 & 0
\end{array}\right] \\
\mathbf{J}_{T_{i 2}} & =\frac{\partial \mathbf{r}_{G_{i 1}}^{(0)}}{\partial \mathbf{q}^{(i)}}=\left[\begin{array}{ccc}
\mathrm{c} \phi_{i} \mathrm{c} \theta_{i}\left(d_{i}-e_{2}\right) & -\mathrm{s} \phi_{i} \mathrm{~s} \theta_{i}\left(d_{i}-e_{2}\right) & \mathrm{c} \phi_{i} \mathrm{~s} \theta_{i} \\
\mathrm{~s} \phi_{i} \mathrm{c} \theta_{i}\left(d_{i}-e_{2}\right) & \mathrm{c} \phi_{i} \mathrm{~s} \theta_{i}\left(d_{i}-e_{2}\right) & \mathrm{s} \phi_{i} \mathrm{~s} \theta_{i} \\
-\mathrm{s} \theta_{i}\left(d_{i}-e_{2}\right) & 0 & \mathrm{c} \theta_{i}
\end{array}\right]
\end{aligned}
$$

From (2.10), the Jacobian matrices of rotation for the links of the $i$-th limb are given by

$$
\mathbf{J}_{R_{i 1}}^{(i)}=\mathbf{J}_{R_{i 2}}^{(i)}=\frac{\partial \mathbf{w}_{i 1}^{(i)}}{\partial \dot{\mathbf{q}}^{(i)}}=\frac{\partial \mathbf{w}_{i 2}^{(i)}}{\partial \dot{\mathbf{q}}^{(i)}}=\left[\begin{array}{ccc}
0 & -\mathrm{s} \theta_{i} & 0 \\
1 & 0 & 0 \\
0 & \mathrm{c} \theta_{i} & 0
\end{array}\right]
$$

The moment of inertia of the cylinder and piston regarding the coordinate system, with the origin at the center of mass $G_{i 1}$ and $G_{i 2}$, respectively, are presented by

$$
\mathbf{I}_{i 1}^{(i)}=\left[\begin{array}{ccc}
I_{x 1} & 0 & 0 \\
0 & I_{y 1} & 0 \\
0 & 0 & I_{z 1}
\end{array}\right] \quad \mathbf{I}_{i 2}^{(i)}=\left[\begin{array}{ccc}
I_{x 2} & 0 & 0 \\
0 & I_{y 2} & 0 \\
0 & 0 & I_{z 2}
\end{array}\right]
$$

According to Khang (2007), the mass matrix of the $i$-th limb is written in the following form

$$
\mathbf{M}^{(i)}\left(\mathbf{q}^{(i)}\right)=\mathbf{J}_{T_{i 1}}^{\mathrm{T}} m_{1} \mathbf{J}_{T_{i 1}}+\mathbf{J}_{T_{i 2}}^{\mathrm{T}} m_{2} \mathbf{J}_{T_{i 2}}+\mathbf{J}_{R_{i 1}^{(i)}}^{\mathrm{T}} \mathbf{I}_{R_{i 1}}^{(i)} \mathbf{J}_{R_{i 1}}^{(i)}+\mathbf{J}_{R_{i 2}}^{(i) T} \mathbf{I}_{R_{i 2}}^{(i)} \mathbf{J}_{R_{i 2}}^{(i)}
$$

Substituting (2.11), (2.12) and (2.13) into (2.14), we calculate and get

$$
\mathbf{M}^{(i)}\left(\mathbf{q}^{(i)}\right)=\left[\begin{array}{lll}
m_{11}^{(i)} & m_{12}^{(i)} & m_{13}^{(i)} \\
m_{21}^{(i)} & m_{22}^{(i)} & m_{23}^{(i)} \\
m_{31}^{(i)} & m_{32}^{(i)} & m_{33}^{(i)}
\end{array}\right]
$$

where

$$
\begin{aligned}
& m_{11}^{(i)}=I_{y_{1}}+I_{y_{2}}+m_{1} e_{1}^{2}+m_{2}\left(d_{i}-e_{2}\right)^{2} \\
& m_{22}^{(i)}=\left[I_{x_{1}}+I_{x_{2}}+m_{1} e_{1}^{2}+m_{2}\left(d_{i}-e_{2}\right)^{2}\right] s^{2} \theta_{i}+\left(I_{z_{1}}+I_{z_{2}}\right) c^{2} \theta_{i} \\
& m_{33}^{(i)}=m_{2} \\
& m_{12}^{(i)}=m_{21}^{(i)}=m_{23}^{(i)}=m_{32}^{(i)}=m_{13}^{(i)}=m_{31}^{(i)}=0
\end{aligned}
$$

The centrifugal and Coriolis matrices (Khang, 2010) of the $i$-th limb are given by

$$
\mathbf{C}^{(i)}\left(\mathbf{q}^{(i)}, \dot{\mathbf{q}}^{(i)}\right)=\frac{\partial \mathbf{M}^{(i)}\left(\mathbf{q}^{(i)}\right)}{\partial \mathbf{q}^{(i)}}\left(\mathbf{I}_{3} \otimes \dot{\mathbf{q}}^{(i)}\right)-\frac{1}{2}\left(\frac{\partial \mathbf{M}^{(i)}\left(\mathbf{q}^{(i)}\right)}{\partial \mathbf{q}^{(i)}}\left(\dot{\mathbf{q}}^{(i)} \otimes \mathbf{I}_{3}\right)\right)^{\mathrm{T}}
$$


Substituting the matrix $\mathbf{M}^{(i)}\left(\mathbf{q}^{(i)}\right)$ from (2.15) into (2.17) and using Maple for calculation, we obtain

$$
\mathbf{C}^{(i)}\left(\mathbf{q}^{(i)}, \dot{\mathbf{q}}^{(i)}\right)=\left[\begin{array}{ccc}
c_{11}^{(i)} & c_{12}^{(i)} & 0 \\
0 & c_{22}^{(i)} & 0 \\
c_{31}^{(i)} & c_{32}^{(i)} & 0
\end{array}\right]
$$

where

$$
\begin{aligned}
c_{11}^{(i)} & =2 m_{2}\left(d_{i}-e_{2}\right) \dot{d}_{i} \\
c_{12}^{(i)} & =-\mathrm{s} \theta_{i} \mathrm{c} \theta_{i}\left[m_{1} e_{1}^{2}+m_{2}\left(d_{i}-e_{2}\right)^{2}+I_{x_{1}}+I_{x_{2}}-I_{z_{1}}-I_{z_{2}}\right] \dot{\phi}_{i} \\
c_{22}^{(i)} & =2 \mathrm{~s} \theta_{i} \mathrm{c} \theta_{i}\left[m_{1} e_{1}^{2}+m_{2}\left(d_{i}-e_{2}\right)^{2}+I_{x_{1}}+I_{x_{2}}-I_{z_{1}}-I_{z_{2}}\right] \dot{\theta}_{i}+2 m_{2}\left(d_{i}-e_{2}\right) s^{2} \theta_{i} \dot{d}_{i} \\
c_{31}^{(i)} & =-m_{2}\left(d_{i}-e_{2}\right) \dot{\theta}_{i} \\
c_{32}^{(i)} & =-m_{2}\left(d_{i}-e_{2}\right) s^{2} \theta_{i} \dot{\phi}_{i} \\
c_{13}^{(i)} & =c_{21}^{(i)}=c_{23}^{(i)}=c_{33}^{(i)}=0
\end{aligned}
$$

The potential energy of the $i$-th limb is expressed by

$$
\Pi^{(i)}=m_{1} \mathbf{g}^{\mathrm{T}} \mathbf{r}_{G_{i 1}}+m_{2} \mathbf{g}^{\mathrm{T}} \mathbf{r}_{G_{i 2}}=m_{1} g\left(a_{i z}+e_{1} \mathrm{c} \theta_{i}\right)+m_{2} g\left[a_{i z}+\left(d_{i}-e_{2}\right) \mathrm{c} \theta_{i}\right]
$$

and

$$
\mathbf{g}^{(i)}\left(\mathbf{q}^{(i)}\right)=\left(\frac{\partial \Pi^{(i)}}{\partial \mathbf{q}^{(i)}}\right)^{\mathrm{T}}=\left[\begin{array}{c}
\left(-m_{1} e_{1}-m_{2} d_{i}+m_{2} e_{2}\right) g \mathrm{~s} \theta_{i} \\
0 \\
m_{2} g \mathrm{c} \theta_{i}
\end{array}\right]
$$

Based on the principle of virtual work, the calculation of the virtual work of non-conservative forces is expressed by

$$
\sum \delta A_{i}^{n p}=\tau_{i} \delta d_{i}-X_{B_{i}} \delta x_{B_{i}}-Y_{B_{i}} \delta y_{B_{i}}-Z_{B_{i}} \delta z_{B_{i}}
$$

The position of the joint $B_{i}$ can be determined by $\mathbf{r}_{B_{i}}=\mathbf{a}_{i}+\mathbf{d}_{i}$. In the coordinate system $\left(O, x_{0}, y_{0}, z_{0}\right)$, we have

$$
\mathbf{r}_{B_{i}}^{(0)}=\mathbf{a}_{i}^{(0)}+\mathbf{A}_{i} \mathbf{d}_{i}^{(i)}=\left[\begin{array}{c}
a_{i x} \\
a_{i y} \\
a_{i z}
\end{array}\right]+\left[\begin{array}{ccc}
\mathrm{c} \phi_{i} \mathrm{c} \theta_{i} & -\mathrm{s} \phi_{i} & \mathrm{c} \phi_{i} \mathrm{~s} \theta_{i} \\
\mathrm{~s} \phi_{i} \mathrm{c} \theta_{i} & \mathrm{c} \phi_{i} & \mathrm{~s} \phi_{i} \mathrm{~s} \theta_{i} \\
-\mathrm{s} \theta_{i} & 0 & \mathrm{c} \theta_{i}
\end{array}\right]\left[\begin{array}{c}
0 \\
0 \\
d_{i}
\end{array}\right]=\left[\begin{array}{c}
a_{i x}+\mathrm{c} \phi_{i} \mathrm{~s} \theta_{i} d_{i} \\
a_{i y}+\mathrm{s} \phi_{i} \mathrm{~s} \theta_{i} d_{i} \\
a_{i z}+\mathrm{c} \theta_{i} d_{i}
\end{array}\right]
$$

Thus, the virtual displacement of the joint $B_{i}$ is given by

$$
\begin{aligned}
& \delta x_{B_{i}}=d_{i} \mathrm{c} \phi_{i} \mathrm{c} \theta_{i} \delta \theta_{i}-d_{i} \mathrm{~s} \phi_{i} \mathrm{~s} \theta_{i} \delta \phi_{i}+\mathrm{c} \phi_{i} \mathrm{~s} \theta_{i} \delta d_{i} \\
& \delta y_{B_{i}}=d_{i} \mathrm{~s} \phi_{i} \mathrm{c} \theta_{i} \delta \theta_{i}+d_{i} \mathrm{c} \phi_{i} \mathrm{~s} \theta_{i} \delta \phi_{i}+\mathrm{s} \phi_{i} \mathrm{~s} \theta_{i} \delta d_{i} \\
& \delta z_{B_{i}}=-d_{i} \mathrm{~s} \theta_{i} \delta \theta_{i}+\mathrm{c} \theta_{i} \delta d_{i}
\end{aligned}
$$

Substituting the components of (2.23) into (2.22), we get

$$
\begin{aligned}
& \sum \delta A_{i}^{n p}=\left(-d_{i} \mathrm{c} \phi_{i} \mathrm{c} \theta_{i} X_{B_{i}}-d_{i} \mathrm{~s} \phi_{i} \mathrm{c} \theta_{i} Y_{B_{i}}+d_{i} \mathrm{~s} \theta_{i} Z_{B_{i}}\right) \delta \theta_{i} \\
& \quad+\left(d_{i} \mathrm{~s} \phi_{i} \mathrm{~s} \theta_{i} X_{B_{i}}-d_{i} \mathrm{c} \phi_{i} \mathrm{~s} \theta_{i} Y_{B_{i}}\right) \delta \phi_{i}+\left(\tau_{i}-X_{B_{i}} \mathrm{c} \phi_{i} \mathrm{~s} \theta_{i}-Y_{B_{i}} \mathrm{~s} \phi_{i} \mathrm{~s} \theta_{i}-Z_{B_{1}} \mathrm{c} \theta_{i}\right) \delta d_{i}
\end{aligned}
$$

Thus, the generalized force corresponding to the conservative force acting on the $i$-th limb is

$$
\mathbf{f}_{i}^{n p}=\left[\begin{array}{c}
-d_{i} \mathrm{c} \phi_{i} \mathrm{c} \theta_{i} X_{B_{i}}-d_{i} \mathrm{~s} \phi_{i} \mathrm{c} \theta_{i} Y_{B_{i}}+d_{i} \mathrm{~s} \theta_{i} Z_{B_{i}} \\
d_{i} \mathrm{~s} \phi_{i} \mathrm{~s} \theta_{i} X_{B_{i}}-d_{i} \mathrm{c} \phi_{i} \mathrm{~s} \theta_{i} Y_{B_{i}} \\
\tau_{i}-X_{B_{i}} \mathrm{c} \phi_{i} \mathrm{~s} \theta_{i}-Y_{B_{i}} \mathrm{~s} \phi_{i} \mathrm{~s} \theta_{i}-Z_{B_{1}} \mathrm{c} \theta_{i}
\end{array}\right]
$$


The system of differential equations describing motion of the $i$-th limb is given in a matrix form

$$
\mathbf{M}^{(i)}\left(\mathbf{q}^{(i)}\right) \ddot{\mathbf{q}}^{(i)}+\mathbf{C}^{(i)}\left(\mathbf{q}^{(i)}, \dot{\mathbf{q}}^{(i)}\right) \dot{\mathbf{q}}^{(i)}+\mathbf{g}^{(i)}\left(\mathbf{q}^{(i)}\right)=\mathbf{f}_{i}^{n p}
$$

We obtain 3 differential equations which are components of equation (2.26) with one value of $i$ $(i=1, \ldots, 6)$. So we have 18 differential equations in total.

\subsection{System of equations for motion of the moving platform}

The mass of the moving platform (in Fig. 3) is denoted by $m_{P}$. The position and orientation of the moving platform are determined by the vector of generalized coordinates $\mathbf{x}=\left[x_{P}, y_{P}, z_{P}, \varphi_{x}, \varphi_{y}, \varphi_{z}\right]^{\mathrm{T}}$.

The forces acting on the moving platform consist of the reaction forces $\mathbf{F}_{i}(i=1, \ldots, 6)$ at the joint $B_{i}$ and the gravity force $m_{P} \mathbf{g}$, where

$$
\begin{aligned}
& \mathbf{F}_{i}^{(0)}=\left[X_{B_{i}}, Y_{B_{i}}, Z_{B_{i}}\right]^{\mathrm{T}} \quad i=1, \ldots, 6 \\
& \mathbf{g}=[0,0,-g]^{\mathrm{T}} \quad g=9.8 \mathrm{~m} / \mathrm{s}^{2}
\end{aligned}
$$

The moment of inertia of the moving platform in the coordinate system $(P, u, v, w)$ is given by

$$
\mathbf{I}_{P}^{(P)}=\left[\begin{array}{ccc}
I_{x 3} & 0 & 0 \\
0 & I_{y 3} & 0 \\
0 & 0 & I_{z 3}
\end{array}\right]
$$

According to Khang (2007), the differential equations of motion are presented in the form of Newton-Euler equations as below

$$
m_{P} \mathbf{a}_{P}=\sum_{i=1}^{6} \mathbf{F}_{i}+m_{P} \mathbf{g}
$$

and

$$
\mathbf{I}_{P} \dot{\boldsymbol{\omega}}_{P}+\boldsymbol{\omega}_{P} \times\left(\mathbf{I}_{P} \boldsymbol{\omega}_{P}\right)=\mathbf{M}_{P}
$$

The angular velocity of the moving platform using the tilde operator is

$$
\widetilde{\boldsymbol{\omega}}_{P}^{(P)}=\dot{\mathbf{A}}_{P} \mathbf{A}_{P}^{\mathrm{T}}=\left[\begin{array}{ccc}
0 & \mathrm{~s} \varphi_{x} \dot{\varphi}_{y}-\mathrm{c} \varphi_{x} \mathrm{c} \varphi_{y} \dot{\varphi}_{z} & \mathrm{c} \phi_{x} \dot{\varphi}_{y}+\mathrm{c} \phi_{x} \mathrm{~s} \phi_{y} \dot{\varphi}_{z} \\
-\mathrm{s} \varphi_{x} \dot{\varphi}_{y}+\mathrm{c} \varphi_{x} \mathrm{c} \varphi_{y} \dot{\varphi}_{z} & 0 & \dot{\varphi}_{x}+\mathrm{s} \phi_{y} \dot{\varphi}_{z} \\
-\mathrm{c} \varphi_{x} \dot{\varphi}_{y}-\mathrm{c} \varphi_{x} \mathrm{~s} \varphi_{y} \dot{\varphi}_{z} & \dot{\varphi}_{x}-\mathrm{s} \varphi_{y} \dot{\varphi}_{z} & 0
\end{array}\right]
$$

then we get

$$
\mathbf{w}_{P}^{(P)}=\left[\begin{array}{c}
\dot{\varphi}_{x}-\mathrm{s} \varphi_{y} \dot{\varphi}_{z} \\
\mathrm{c} \varphi_{x} \dot{\varphi}_{y}+\mathrm{c} \varphi_{x} \mathrm{~s} \varphi_{y} \dot{\varphi}_{z} \\
-\mathrm{s} \varphi_{x} \dot{\varphi}_{y}+\mathrm{c} \varphi_{x} \mathrm{c} \varphi_{y} \dot{\varphi}_{z}
\end{array}\right]
$$

and the angular acceleration is

$$
\boldsymbol{\alpha}_{P}^{(P)}=\frac{d \mathbf{w}_{P}^{(P)}}{d t}=\left[\begin{array}{c}
\ddot{\varphi}_{x}-\mathrm{s} \varphi_{y} \ddot{\varphi}_{z}-\mathrm{c} \varphi_{y} \dot{\varphi}_{y} \dot{\varphi}_{z} \\
\mathrm{c} \varphi_{x} \ddot{\varphi}_{y}-\mathrm{s} \varphi_{x} \mathrm{c} \varphi_{y} \ddot{\varphi}_{z}-\mathrm{s} \varphi_{x} \dot{\varphi}_{x} \dot{\varphi}_{y}+\mathrm{c} \varphi_{x} \mathrm{c} \varphi_{y} \dot{\varphi}_{x} \dot{\varphi}_{z}-\mathrm{s} \varphi_{x} \mathrm{~s} \varphi_{y} \dot{\varphi}_{y} \dot{\varphi}_{z} \\
-\mathrm{s} \varphi_{x} \ddot{\varphi}_{y}+\mathrm{c} \varphi_{x} \mathrm{c} \varphi_{y} \ddot{\varphi}_{z}-\mathrm{c} \varphi_{x} \dot{\varphi}_{x} \dot{\varphi}_{y}-\mathrm{s} \varphi_{x} \mathrm{c} \varphi_{y} \dot{\varphi}_{x} \dot{\varphi}_{z}-\mathrm{c} \varphi_{x} \mathrm{~s} \varphi_{y} \dot{\varphi}_{y} \dot{\varphi}_{z}
\end{array}\right]
$$

The velocity and acceleration of the point $P$ are given by

$$
\mathbf{v}_{P}^{(0)}=\frac{d \mathbf{r}_{P}^{(0)}}{d t}=\left[\dot{x}_{P}, \dot{y}_{P}, \dot{z}_{P}\right]^{\mathrm{T}} \quad \mathbf{a}_{P}^{(0)}=\frac{d \mathbf{v}_{P}^{(0)}}{d t}=\left[\ddot{x}_{P}, \ddot{y}_{P}, \ddot{z}_{P}\right]^{\mathrm{T}}
$$


The moment of reaction forces about point $P$ on the moving platform is presented by

$$
\mathbf{M}_{P}=\sum_{i=1}^{6} \mathbf{b}_{i} \times \mathbf{F}_{i}
$$

and in the coordinate system $(P, u, v, w)$, it can be written in the algebraic matrix form

$$
\mathbf{M}_{P}^{(P)}=\sum_{i=1}^{6} \widetilde{\mathbf{b}}_{i}^{(P)} \mathbf{F}_{i}^{(P)}
$$

where $\mathbf{b}_{i}^{(P)}=\left[b_{i u}, b_{i v}, b_{i w}\right]^{\mathrm{T}}, \mathbf{F}_{i}^{(P)}=\mathbf{A}_{P}^{\mathrm{T}} \mathbf{F}_{i}^{(0)}$ and leads to the tilde matrix

$$
\widetilde{\mathbf{b}}_{i}^{(P)}=\left[\begin{array}{ccc}
0 & -b_{i w} & b_{i v} \\
b_{i w} & 0 & -b_{i u} \\
-b_{i v} & b_{i u} & 0
\end{array}\right]
$$

We can rewrite equation (2.27) in the global coordinate system $\left(O, x_{0}, y_{0}, z_{0}\right)$ and equation $(2.28)$ in the local coordinate system $(P, u, v, w)$ as below

$$
m_{P} \mathbf{a}_{P}^{(0)}=\sum_{i=1}^{6} \mathbf{F}_{i}^{(0)}-m_{P} \mathbf{g}
$$

and

$$
\mathbf{I}_{P}^{(P)} \boldsymbol{\alpha}_{P}^{(p)}+\widetilde{\boldsymbol{\omega}}_{P}^{(P)}\left(\mathbf{I}_{P}^{(P)} \boldsymbol{\omega}_{P}^{(P)}\right)=\mathbf{M}_{P}^{(P)}
$$

Considering components of equations (2.34) and (2.35), we obtain six differential equations for motion of the moving platform

$$
\begin{aligned}
& m_{P} \ddot{x}_{P}=X_{B_{1}}+X_{B_{2}}+X_{B_{3}}+X_{B_{4}}+X_{B_{5}}+X_{B_{6}} \\
& m_{P} \ddot{y}_{P}=Y_{B_{1}}+Y_{B_{2}}+Y_{B_{3}}+Y_{B_{4}}+Y_{B_{5}}+Y_{B_{6}} \\
& m_{P} \ddot{z}_{P}=Z_{B_{1}}+Z_{B_{2}}+Z_{B_{3}}+Z_{B_{4}}+Z_{B_{5}}+Z_{B_{6}}-m_{P} g
\end{aligned}
$$

and

$$
\begin{aligned}
& I_{x 3}\left(\ddot{\varphi}_{x}-\mathrm{s} \varphi_{y} \ddot{\varphi}_{z}-\mathrm{c} \varphi_{y} \dot{\varphi}_{y} \dot{\varphi}_{z}\right)+I_{y 3}\left(\mathrm{~s} \varphi_{x} \dot{\varphi}_{y}-\mathrm{c} \varphi_{x} \mathrm{c} \varphi_{y} \dot{\varphi}_{z}\right)\left(\mathrm{c} \varphi_{x} \dot{\varphi}_{y}+\mathrm{c} \varphi_{x} \mathrm{~s} \varphi_{y} \dot{\varphi}_{z}\right) \\
& \quad+I_{z 3}\left(\mathrm{c} \varphi_{x} \dot{\varphi}_{y}+\mathrm{c} \varphi_{x} \mathrm{~s} \varphi_{y} \dot{\varphi}_{z}\right)\left(-\mathrm{s} \varphi_{x} \dot{\varphi}_{y}+\mathrm{c} \varphi_{x} \mathrm{c} \varphi_{y} \dot{\varphi}_{z}\right) \\
& \quad=\sum_{i=1}^{6}\left[-b_{i w}\left(-\mathrm{s} \varphi_{z} \mathrm{c} \varphi_{x}+\mathrm{c} \varphi_{z} \mathrm{~s} \varphi_{y} \mathrm{~s} \varphi_{x}\right)-b_{i v}\left(-\mathrm{s} \varphi_{z} \mathrm{~s} \varphi_{x}-\mathrm{c} \varphi_{z} \mathrm{~s} \varphi_{y} \mathrm{c} \varphi_{x}\right)\right] X_{B_{i}} \\
& \quad+\sum_{i=1}^{6}\left[-b_{i w}\left(\mathrm{c} \varphi_{z} \mathrm{c} \varphi_{x}+\mathrm{s} \varphi_{z} \mathrm{~s} \varphi_{y} \mathrm{~s} \varphi_{x}\right)-b_{i v}\left(\mathrm{c} \varphi_{z} \mathrm{~s} \varphi_{x}-\mathrm{s} \varphi_{z} \mathrm{~s} \varphi_{y} \mathrm{c} \varphi_{x}\right)\right] Y_{B_{i}} \\
& \quad+\sum_{i=1}^{6}\left[-b_{i w} \mathrm{c} \varphi_{y} \mathrm{~s} \varphi_{x}+b_{i v} \mathrm{c} \varphi_{y} \mathrm{c} \varphi_{x}\right] Z_{B_{i}} \\
& \quad+I_{y 3}\left(\mathrm{c} \varphi_{x} \ddot{\varphi}_{y}-\mathrm{s} \varphi_{x} \mathrm{c} \varphi_{y} \ddot{\varphi}_{z}-\mathrm{s} \varphi_{x} \dot{\varphi}_{x} \dot{\varphi}_{y}+\mathrm{c} \varphi_{x} \mathrm{c} \varphi_{y} \dot{\varphi}_{x} \dot{\varphi}_{z}-\mathrm{s} \varphi_{x} \mathrm{~s} \varphi_{y} \dot{\varphi}_{y} \dot{\varphi}_{z}\right) \\
& \quad=\sum_{i=1}^{6}\left[b_{i w} \mathrm{c} \varphi_{z} \mathrm{c} \varphi_{y}-b_{i u}\left(\mathrm{~s} \varphi_{z} \mathrm{~s} \varphi_{x}+\mathrm{c} \varphi_{z} \mathrm{~s} \varphi_{y} \mathrm{c} \varphi_{x}\right)\right] X_{B_{i}} \\
& \quad+\sum_{i=1}^{6}\left[b_{i w} \mathrm{~s} \varphi_{z} \mathrm{c} \varphi_{x}-b_{i u}\left(-\mathrm{c} \varphi_{z} \mathrm{~s} \varphi_{x}+\mathrm{s} \varphi_{z} \mathrm{~s} \varphi_{y} \mathrm{c} \varphi_{x}\right)\right] Y_{B_{i}}+\sum_{i=1}^{6}\left[-b_{i w} \mathrm{~s} \varphi_{y}-b_{i u} \mathrm{c} \varphi_{y} \mathrm{c} \varphi_{x}\right] Z_{B_{i}}
\end{aligned}
$$




$$
\begin{aligned}
& I_{x 3}\left(\dot{\varphi}_{x}-\mathrm{s} \varphi_{y} \dot{\varphi}_{z}\right)\left(-\mathrm{c} \varphi_{x} \dot{\varphi}_{y}-\mathrm{c} \varphi_{x} \mathrm{~s} \varphi_{y} \dot{\varphi}_{z}\right)+I_{y 3}\left(\dot{\varphi}_{x}-\mathrm{s} \varphi_{y} \dot{\varphi}_{z}\right)\left(\mathrm{c} \varphi_{x} \dot{\varphi}_{y}+\mathrm{c} \varphi_{x} \mathrm{~s} \varphi_{y} \dot{\varphi}_{z}\right) \\
& \quad+I_{z 3}\left(-\mathrm{s} \varphi_{x} \ddot{\varphi}_{y}+\mathrm{c} \varphi_{x} \mathrm{c} \varphi_{y} \ddot{\varphi}_{z}-\mathrm{c} \varphi_{x} \dot{\varphi}_{x} \dot{\varphi}_{y}-\mathrm{s} \varphi_{x} \mathrm{c} \varphi_{y} \dot{\varphi}_{x} \dot{\varphi}_{z}-\mathrm{c} \varphi_{x} \mathrm{~s} \varphi_{y} \dot{\varphi}_{y} \dot{\varphi}_{z}\right) \\
& \quad=\sum_{i=1}^{6}\left[-b_{i v} \mathrm{c} \varphi_{z} \mathrm{c} \varphi_{y}-b_{i u}\left(\mathrm{~s} \varphi_{z} \mathrm{c} \varphi_{x}-\mathrm{c} \varphi_{z} \mathrm{~s} \varphi_{y} \mathrm{~s} \varphi_{x}\right)\right] X_{B_{i}} \\
& \quad+\sum_{i=1}^{6}\left[-b_{i v} \mathrm{~s} \varphi_{z} \mathrm{c} \varphi_{y}+b_{i u}\left(\mathrm{c} \varphi_{z} \mathrm{c} \varphi_{x}+\mathrm{s} \varphi_{z} \mathrm{~s} \varphi_{y} \mathrm{~s} \varphi_{x}\right)\right] Y_{B_{i}}+\sum_{i=1}^{6}\left[b_{i v} \mathrm{~s} \varphi_{y}+b_{i u} \mathrm{c} \varphi_{y} \mathrm{~s} \varphi_{x}\right] Z_{B_{i}}
\end{aligned}
$$

where $i=1,2, \ldots, 6$.

Thus, we obtain 18 differential equations from (2.26) for motion of six limbs, 6 differential equations (2.36) and (2.37) for the moving platform, and 18 constraint equations taken from (2.7). In total, we obtain a system of 42 differential-algebraic equations to describe the overall Stewart-Gough platform motion.

Briefly, the 24 differential equations, which are taken from (2.26), (2.36) and (2.37), can be abbreviated in a matrix form

$$
\mathbf{M}(\mathbf{s}) \ddot{\mathbf{s}}+\mathbf{v}(\mathbf{s} \dot{\mathbf{s}})+\mathbf{g}(\mathbf{s})=\boldsymbol{\tau}-\mathbf{J}_{s}^{\mathrm{T}}(\mathbf{s}) \boldsymbol{\lambda}
$$

and the 18 constraint equations can be abbreviated in the form

$$
\mathbf{f}(\mathbf{s})=\mathbf{0}
$$

where $\boldsymbol{\lambda}$ is a vector of constraint forces acting on the moving platform

$$
\boldsymbol{\lambda}=\left[X_{B 1}, Y_{B 1}, Z_{B 1}, \ldots, X_{B 6}, Y_{B 6}, Z_{B 6}\right]^{\mathrm{T}}
$$

$\tau$ is the vector of input forces exerted on the actuated joints

$$
\boldsymbol{\tau}=\left[\boldsymbol{\tau}_{a}^{\mathrm{T}}, \boldsymbol{\tau}_{z}^{\mathrm{T}}\right]^{\mathrm{T}} \quad \boldsymbol{\tau} \in \mathbb{R}^{n_{s}}
$$

$\mathbf{s}$ is a vector of minimum generalized coordinates used for determining the position and orientation of the Stewart-Gough platform

$$
\mathbf{s}=\left[\mathbf{q}_{a}^{\mathrm{T}}, \mathbf{q}_{p}^{\mathrm{T}}, \mathbf{x}^{\mathrm{T}}\right]^{\mathrm{T}}=\left[\mathbf{q}_{a}^{\mathrm{T}}, \mathbf{z}^{\mathrm{T}} t\right]^{\mathrm{T}} \quad, \mathbf{s} \in \mathbb{R}^{n_{s}} \quad n_{s}=n_{a}+n_{z}
$$

$\tau_{a}=\left[\tau_{1}, \tau_{2}, \tau_{3}, \tau_{4}, \tau_{5}, \tau_{6}\right]^{\mathrm{T}}, \boldsymbol{\tau}_{a} \in \mathbb{R}^{n_{a}}$ is a vector of input forces exerted on the actuated joints, $\tau_{r}=[0,0, \ldots, 0]^{\mathrm{T}}, \boldsymbol{\tau}_{r} \in \mathbb{R}^{n_{z}}, \mathbf{q}_{a}=\left[d_{1}, d_{2}, d_{3}, d_{4}, d_{5}, d_{6}\right]^{\mathrm{T}}$ is a vector of generalized coordinates of the length of limbs, $\mathbf{q}_{p}=\left[\theta_{1}, \theta_{2}, \theta_{3}, \theta_{4}, \theta_{5}, \theta_{6}, \phi_{1}, \phi_{2}, \phi_{3}, \phi_{4}, \phi_{5}, \phi_{6}\right]^{\mathrm{T}}$ is a vector of generalized coordinates of the limb rotation, $\mathbf{x}=\left[x_{P}, y_{P}, z_{P}, \varphi_{x}, \varphi_{y}, \varphi_{z}\right]^{\mathrm{T}}$ is a six-dimensional vector used for specification of the global position and orientation of the moving platform in the coordinate system $\left.\left(O, x_{0}, y_{0}, z_{0}\right), \mathbf{z}=\left[\mathbf{q}_{p}^{\mathrm{T}}, \mathbf{x}^{\mathrm{T}}\right]^{\mathrm{T}}, \mathbf{z} \in \mathbb{R}^{n_{z}}\right]$ and

$$
\begin{array}{llrl}
\mathbf{M}(\mathbf{s}) \in \mathbb{R}^{n_{s} \times n_{s}} & \mathbf{f} \in \mathbb{R}^{r} \quad \mathbf{J}_{s}^{\mathrm{T}}(\mathbf{s}) \in \mathbb{R}^{n_{s} \times r} \quad \boldsymbol{\lambda} \in \mathbb{R}^{r} \\
\mathbf{J}_{s}=\frac{\partial \mathbf{f}}{\partial \mathbf{s}} & \mathbf{g}(\mathbf{s}) \in \mathbb{R}^{n_{s}} & \mathbf{v}(\mathbf{s}, \dot{\mathbf{s}}) \in \mathbb{R}^{n_{s}} &
\end{array}
$$

This system of equations contains 42 equations with 42 variables (including 18 constraint forces in (2.40) and 24 generalized coordinates in (2.42)), so it is possible to solve and find the solution.

\section{Solving the system of abbreviated differential equations for the inverse dynamics problem}

The problem of inverse dynamics can be described as follows: given the desired trajectory of the moving platform motion $\mathbf{x}=\mathbf{x}(t), \mathbf{x} \in \mathbb{R}^{m}$ and constraint equations $\mathbf{f}(\mathbf{x}, \mathbf{q})=\mathbf{0}$, the task is 
to determine the vector of input forces $\tau$ exerted on the actuated joints. An idea proposed to solve the problem is based on the elimination of the generalized coordinates $s$ and the vector of constraint forces $\boldsymbol{\lambda}$, transforming differential/algebraic equations (2.38) and (2.39) into ordinary differential equations with variables representing the components of the vector $\mathbf{q}_{a}$. The number of equations is equal to the number of degrees of freedom of the Stewart-Gough platform (see Khang, 2013).

It is possible to rewrite (2.39) in the following form

$$
\mathbf{f}(\mathbf{s})=\mathbf{f}\left(\mathbf{q}_{a}, \mathbf{z}\right)=\mathbf{0} \quad \mathbf{f} \in \mathbb{R}^{r} \quad \mathbf{z} \in \mathbb{R}^{n_{z}} \quad \mathbf{q}_{a} \in \mathbb{R}^{n_{a}}
$$

Supposing that the number of generalized coordinates is equal to the number of additional constraint equations $r=n_{z}$. From equation (3.1) we have

$$
\delta \mathbf{f}=\frac{\partial \mathbf{f}}{\partial \mathbf{s}} \delta \mathbf{s}=\mathbf{J}_{s}(\mathbf{s}) \delta \mathbf{s}=\mathbf{0}
$$

which results in

$$
\mathbf{J}_{s}(\mathbf{s}) \delta \mathbf{s}=\mathbf{J}_{a}(\mathbf{s}) \delta \mathbf{q}_{a}+\mathbf{J}_{z}(\mathbf{s}) \delta \mathbf{z}=\mathbf{0}
$$

Rewriting equation (2.38), we obtain

$$
\mathbf{J}_{s}^{\mathrm{T}}(\mathbf{s}) \boldsymbol{\lambda}=\mathbf{M}(\mathbf{s}) \ddot{\mathbf{s}}+\mathbf{v}(\mathbf{s}, \dot{\mathbf{s}})+\mathbf{g}(\mathbf{s})-\boldsymbol{\tau}
$$

Transposing both sides of equation (3.4) and multiplying by $\delta \mathbf{s}$, we obtain

$$
\lambda^{\mathrm{T}} \mathbf{J}_{s} \delta \mathbf{s}=[\mathbf{M}(\mathbf{s}) \ddot{\mathbf{s}}+\mathbf{v}(\mathbf{s}, \dot{\mathbf{s}})+\mathbf{g}(\mathbf{s})-\boldsymbol{\tau}]^{\mathrm{T}} \delta \mathbf{s}
$$

Considering equation (3.3), we have

$$
[\mathbf{M}(\mathbf{s}) \ddot{\mathbf{s}}+\mathbf{v}(\mathbf{s}, \dot{\mathbf{s}})+\mathbf{g}(\mathbf{s})-\boldsymbol{\tau}]^{\mathrm{T}} \delta \mathbf{s}=\mathbf{0}^{\mathrm{T}}
$$

Moreover, from equation (3.3), we obtain

$$
\delta \mathbf{z}=-\mathbf{J}_{z}^{-1}(\mathbf{s}) \mathbf{J}_{a}(\mathbf{s}) \delta \mathbf{q}_{a}
$$

so

$$
\delta \mathbf{s}=\left[\begin{array}{c}
\delta \mathbf{q}_{a} \\
\delta \mathbf{z}
\end{array}\right]=\left[\begin{array}{c}
\mathbf{E}_{n_{a}} \\
-\mathbf{J}_{z}^{-1}(\mathbf{s}) \mathbf{J}_{a}(\mathbf{s})
\end{array}\right] \delta \mathbf{q}_{a}
$$

where $\mathbf{E}_{n_{a}}$ is the identity matrix.

Denoting

$$
\mathbf{R}(\mathbf{s})=\left[\begin{array}{c}
\mathbf{E}_{n_{a}} \\
-\mathbf{J}_{z}^{-1}(\mathbf{s}) \mathbf{J}_{a}(\mathbf{s})
\end{array}\right]
$$

equation (3.8) can be expressed as

$$
\delta \mathbf{s}=\mathbf{R}(\mathbf{s}) \delta \mathbf{q}_{a}
$$

We divided both sides of equation (3.10) by $d t$ and then get

$$
\dot{\mathbf{s}}=\mathbf{R}(\mathbf{s}) \dot{\mathbf{q}}_{a}
$$


Substituting (3.11) into (3.6), we get

$$
[\mathbf{M}(\mathbf{s}) \ddot{\mathbf{s}}+\mathbf{v}(\mathbf{s} \dot{\mathbf{s}})+\mathbf{g}(\mathbf{s})-\boldsymbol{\tau}]^{\mathrm{T}} \mathbf{R}(\mathbf{s}) \delta \mathbf{q}_{a}=\mathbf{0}^{\mathrm{T}}
$$

Considering the vector $\delta \mathbf{q}=\left[\delta q_{1}^{a}, \delta q_{2}^{a}, \ldots, \delta q_{n_{a}}^{q}\right]^{\mathrm{T}}$ is not a zero vector in general, from (3.12), we can write

$$
\mathbf{R}^{\mathrm{T}}(\mathbf{s})[\mathbf{M}(\mathbf{s}) \ddot{\mathbf{s}}+\mathbf{v}(\mathbf{s}, \dot{\mathbf{s}})+\mathbf{g}(\mathbf{s})-\boldsymbol{\tau}]=\mathbf{0}
$$

Equation (3.13) can be presented in the form

$$
\mathbf{R}^{\mathrm{T}}(\mathbf{s}) \boldsymbol{\tau}=\mathbf{R}^{\mathrm{T}}(\mathbf{s})[\mathbf{M}(\mathbf{s}) \ddot{\mathbf{s}}+\mathbf{v}(\mathbf{s}, \dot{\mathbf{s}})+\mathbf{g}(\mathbf{s})]
$$

Substituting (2.41) and (3.9) into the left-hand side of equation (3.14), we get

$$
\mathbf{R}^{\mathrm{T}}(\mathbf{s}) \boldsymbol{\tau}=\left[\mathbf{E}_{n a},-\left(\mathbf{J}_{z}^{-1}(\mathbf{s}) \mathbf{J}_{a}(\mathbf{s})\right)^{\mathrm{T}}\right]\left[\begin{array}{c}
\boldsymbol{\tau}_{a} \\
\boldsymbol{\tau}_{z}
\end{array}\right]=\boldsymbol{\tau}_{a}-\left[\mathbf{J}_{z}^{-1}(\mathbf{s}) \mathbf{J}_{a}(\mathbf{s})\right]^{\mathrm{T}} \boldsymbol{\tau}_{z}
$$

Substituting (3.15) into (3.14), we arrive at

$$
\boldsymbol{\tau}_{a}=\mathbf{R}^{\mathrm{T}}(\mathbf{s})[\mathbf{M}(\mathbf{s}) \ddot{\mathbf{s}}+\mathbf{v}(\mathbf{s}, \dot{\mathbf{s}})+\mathbf{g}(\mathbf{s})]+\left[\mathbf{J}_{z}^{-1}(\mathbf{s}) \mathbf{J}_{a}(\mathbf{s})\right]^{\mathrm{T}} \boldsymbol{\tau}_{z}
$$

In brief, the solution of the inverse dynamics problem based on the system of abbreviated differential equations with minimum generalized coordinates can be achieved by following the steps below.

Step 1: Given $\mathbf{x}(t)$ and $\mathbf{f}(\mathbf{x q})=\mathbf{0}$, determine $\mathbf{s}(t), \dot{\mathbf{s}}(t), \ddot{\mathbf{s}}(t)$, solving the problem of inverse kinematics.

Step 2: Determine the matrices $\mathbf{J}_{z}(\mathbf{s}), \mathbf{J}_{a}(\mathbf{s}), \mathbf{J}_{z}^{-1}(\mathbf{s}), \mathbf{R}(\mathbf{s}), \mathbf{M}(\mathbf{s}), \mathbf{v}(\mathbf{s}, \dot{\mathbf{s}}), \mathbf{g}(\mathbf{s})$.

Step 3: Determine the vector of input forces $\boldsymbol{\tau}_{a}$ using equation (3.16).

\section{Numerical example}

According to Tsai (2000), the value of parameters used for simulations was obtained by Wang and Gosselin (1998). The positions of joints $A_{i}(i=1, \ldots, 6)$ in the global coordinate system $\left(O, x_{0}, y_{0}, z_{0}\right)$ can be determined by the algebraic vectors $\mathbf{a}_{i}[\mathrm{~m}]$ and $\mathbf{b}_{i}[\mathrm{~m}]$

$$
\begin{array}{lll}
\mathbf{a}_{1}=[-2.120,1.374,0]^{\mathrm{T}} & \mathbf{a}_{2}=[-2.380,1.224,0]^{\mathrm{T}} \\
\mathbf{a}_{3}=[-2.380,-1.224,0]^{\mathrm{T}} & \mathbf{a}_{4}=[-2.120,-1.374,0]^{\mathrm{T}} \\
\mathbf{a}_{5}=[0,-0.15,0]^{\mathrm{T}} & \mathbf{a}_{6}=[-2.380,-1.224,0]^{\mathrm{T}} \\
\mathbf{b}_{1}=[0.170,0.595,-0.4]^{\mathrm{T}} & \mathbf{b}_{2}=[-0.6,0.15,-0.4]^{\mathrm{T}} \\
\mathbf{b}_{3}=[-0.6,-0.15,-0.4]^{\mathrm{T}} & \mathbf{b}_{4}=[0.170,-0.595,-0.4]^{\mathrm{T}} \\
\mathbf{b}_{5}=[0.43,-0.445,-0.4]^{\mathrm{T}} & \mathbf{b}_{6}=[0.43,0.445,-0.4]^{\mathrm{T}}
\end{array}
$$

The mass of the moving platform is $m_{P}=1.5 \mathrm{~kg}$.

The mass and dimension of links 1 and 2 for each limb are given as follows: $m_{1}=0.1 \mathrm{~kg}$, $e_{1}=0.5 \mathrm{~m}$ and $m_{2}=0.1 \mathrm{~kg}, e_{2}=0.5 \mathrm{~m}$. 
The moments of inertia of the moving platform and links are (in $\left[\mathrm{kg} / \mathrm{m}^{2}\right]$ )

$$
\begin{aligned}
& \mathbf{I}_{P}^{(P)}=\left[\begin{array}{ccc}
0.08 & 0 & 0 \\
0 & 0.08 & 0 \\
0 & 0 & 0.08
\end{array}\right] \quad \mathbf{I}_{i 1}^{(i)}=\left[\begin{array}{ccc}
0.00625 & 0 & 0 \\
0 & 0.00625 & 0 \\
0 & 0 & 0
\end{array}\right] \\
& \mathbf{I}_{i 2}^{(i)}=\left[\begin{array}{ccc}
0.00625 & 0 & 0 \\
0 & 0.00625 & 0 \\
0 & 0 & 0
\end{array}\right]
\end{aligned}
$$

The equations and graphs for the desired trajectory describing motion of the center of mass of the moving platform and the simulation results of the input forces for several cases are presented in Table 1. The simulation was performed using Maple software.

The simulation results for cases 1 and 2 matches the results by Tsai (2000).

Table 1. The simulation $\left(\mathbf{r}_{P}=\left[x_{P}, y_{P}, z_{P}\right]^{\mathrm{T}}\right.$ in $[\mathrm{m}], \omega$ in $\left.[\mathrm{rad} / \mathrm{s}]\right)$

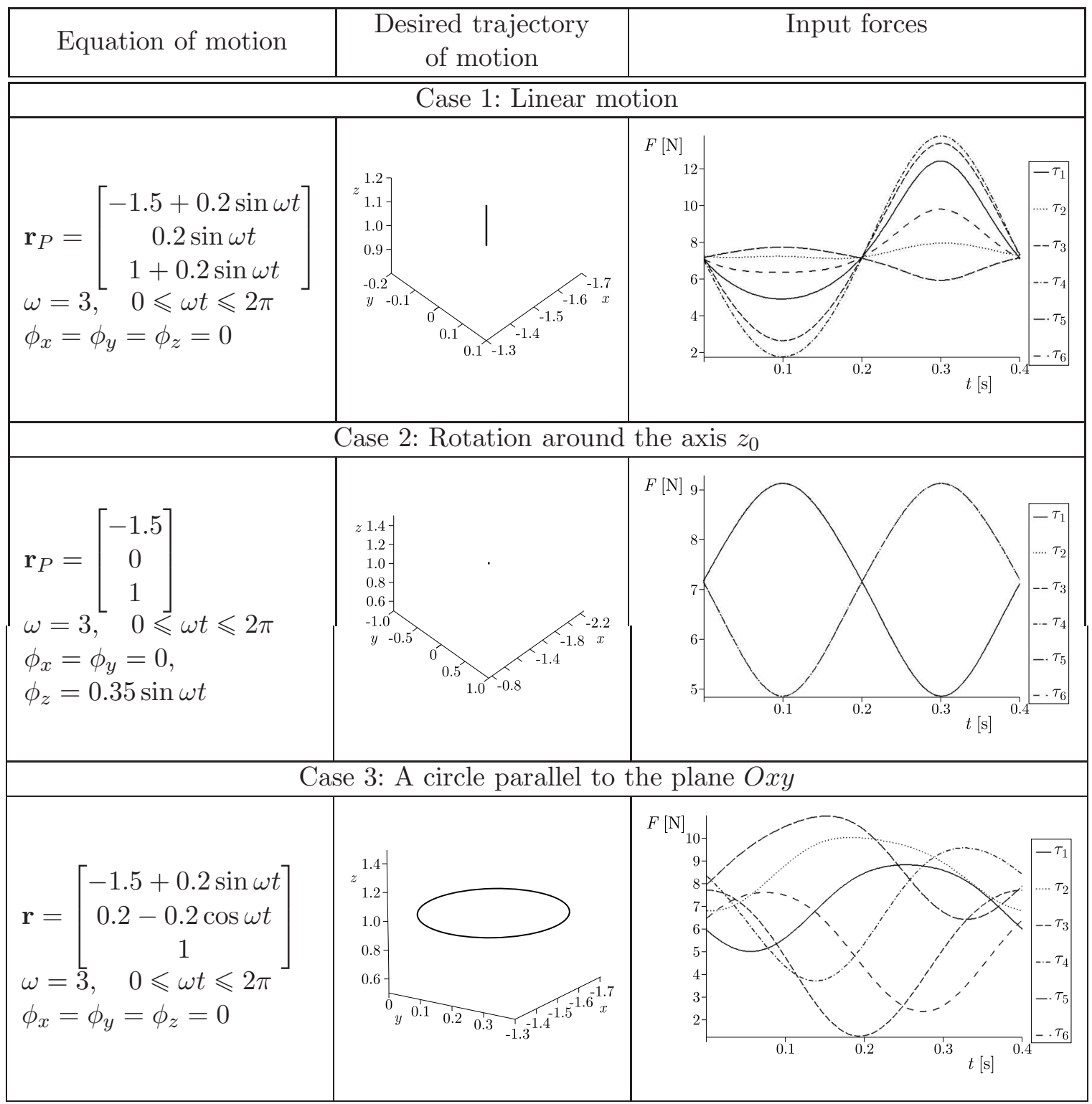




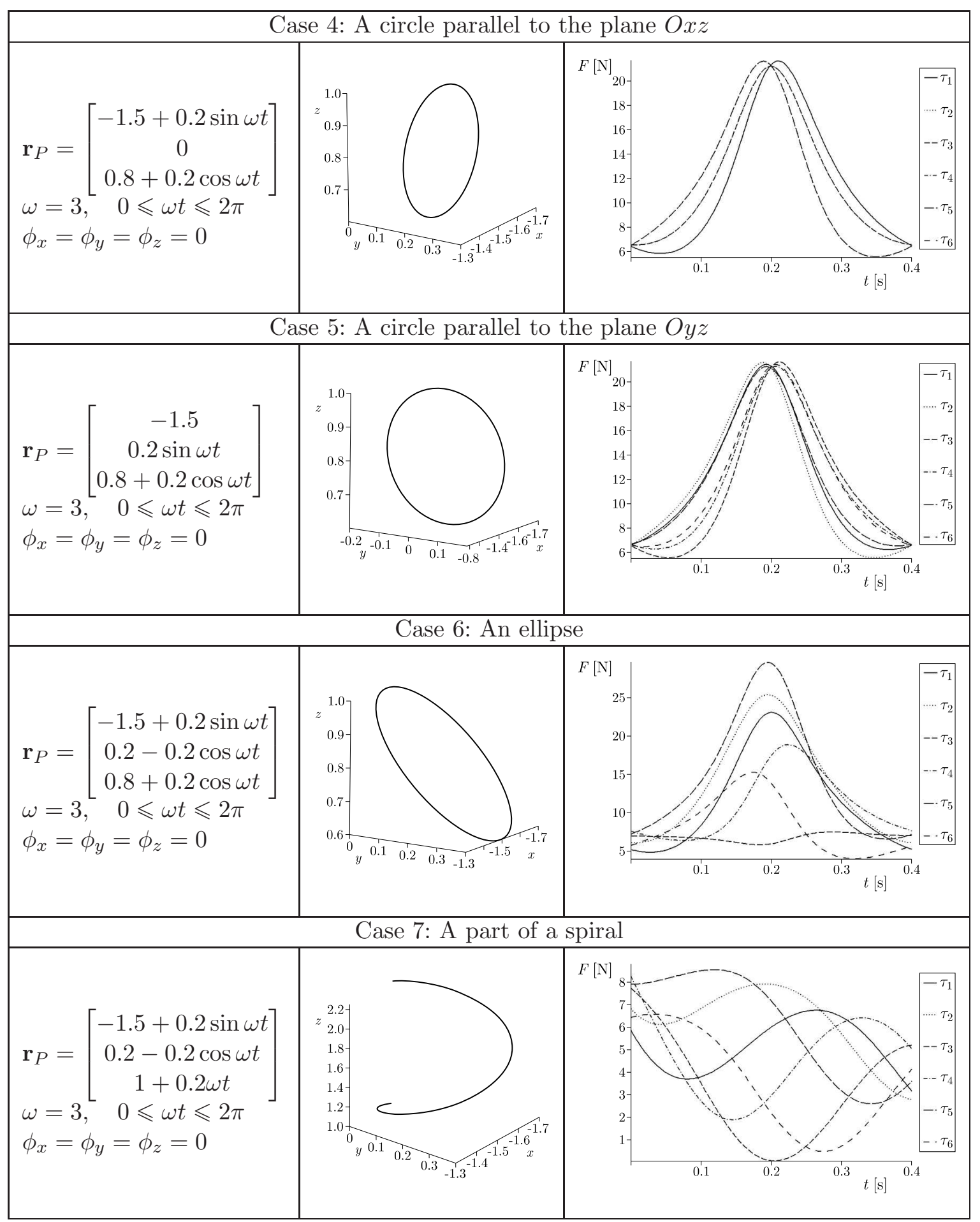

\section{Conclusion}

The Stewart-Gough platform is used extensively in mechanical engineering and medical biology practice. Therefore, studying the dynamics and control of the Stewart-Gough platform is very important. In this article, the substructure technique method and a matrix of Lagrange equations were used to establish the system of equations of motion of the Stewart-Gough platform, and the inverse dynamics problem of the Stewart-Gough platform was solved. The simulation results for cases 1 and 2, which match with the results by Tsai (2000), verify the correctness 
of the method used in this article. With the use of the substructure technique, the problem of determining the equations of motion for a complex spatial structure becomes clear and realizable. The computational method for the Stewart-Gough platform can be applied to modeling of other parallel platforms.

\section{References}

1. Angeles J., 1997, Fundamentals of Robotic Mechanical Systems, Springer Verlag, New York, N.Y.

2. Codourey A., Burdet E., 1997, A body-oriented method for finding a linear form of the dynamic equation of fully parallel robots, Proceedings of the 1997 IEEE International Conference on Robotics and Automation, Albuquerque, New Mexico, 1612-1618

3. Do W., YANG D., 1988, Inverse dynamic analysis and simulation of a platform type of robot, Journal of Robotic Systems, 5, 3, 209-227

4. Etemadi Zanganeh K., Sinatra R., Angeles J., 1997, Kinematics and dynamics of a six-degree-of-freedom parallel manipulator with revolute legs, Robotica, 15, 385-394

5. Guglielmetti P., Longchamp R., 1994, A closed form inverse dynamics model of the delta parallel robot, Proceedings of International Federation of Automatic Control Conference on Robot Control, 39-44

6. Khang N.V., 2007, Dynamics of Multibody Systems, Publishing Company of Science and Technology, Ha Noi

7. Khang N.V., 2010, Consistent definition of partial derivatives of matrix functions in dynamics of mechanical systems, Mechanism and Machine Theory, 45, 981-988

8. Khang N.V., 2011, Kronecker product and a new matrix form of Lagrangian equations with multipliers for constrained multibody systems, Mechanics Research Communications, 38, 294-299

9. Khang N.V., 2013, Inverse dynamics of constrained multibody systems, Vietnam Journal of Mechanics, VAST, $\mathbf{3 5}$

10. Lebret G., Liu K., Lewis F., 1993, Dynamic analysis and control of a stewart platform manipulator, Journal of Robotic Systems, 10, 5, 629-655

11. Miller K., 1995, Experimental verification of modeling of delta robot dynamics by application of Hamilton's principle, Proceedings of the 1995 IEEE International Conference on Robotics and Automation, 532-537

12. Miller K., Clavel R., 1992, The Lagrange-based model of delta-4 robot dynamics, Robotersysteme, 8, 49-54

13. Pang H., Shahingpoor M., 1994, Inverse dynamics of a parallel manipulator, Journal of Robotic Systems, 11, 8, 693-702

14. Tsai K., Kohli D., 1990, Modified Newton-Euler computational scheme for dynamic analysis and simulation of parallel manipulators with applications to configuration based on R-L actuators, Proceedings of ASME Design Engineering Technical Conferences, 24, 111-117

15. Tsai L.W., 2000, Solving the inverse dynamics of a Stewart-Gough manipulator by the principle of virtual work, Journal of Mechanical Design, 122, 3-9

16. Wang J., Gosselin C.M., 1997, Dynamic analysis of spatial four degree-of-freedom parallel manipulators, Proceedings the 1997 ASME Design Engineering Technical Conferences, Sacramento, CA, Paper No.DETC97/DAC3759

17. WAng J., Gosselin C., 1998, A new approach for the dynamic analysis of parallel manipulators, Multibody System Dynamics, 2, 317-334

18. Zhang C.D., Song S.M., 1993, An efficient method for inverse dynamics of manipulators based on the virtual work principle, Journal of Robotic Systems, 10, 5, 605-627 